\title{
Post Embedding Problem Is Not Primitive Recursive, With Applications To Channel Systems ${ }^{\star}$
}

\author{
P. Chambart and Ph. Schnoebelen \\ LSV, ENS Cachan, CNRS \\ 61, av. Pdt. Wilson, F-94230 Cachan, France \\ email: \{chambart|phs\}@1sv.ens-cachan.fr
}

\begin{abstract}
We introduce PEP, the Post Embedding Problem, a variant of PCP where one compares strings with the subword relation, and PEP ${ }^{\text {reg }}$, a further variant where solutions are constrained and must belong to a given regular language. PEPreg is decidable but not primitive recursive. This entails the decidability of reachability for unidirectional systems with one reliable and one lossy channel.
\end{abstract}

Keywords: Post correspondence problem; Lossy channel systems; Higman's Lemma.

\section{Introduction}

Post correspondence problem, or shortly PCP, can be stated as the question whether two morphisms $u, v: \Sigma^{*} \rightarrow \Gamma^{*}$ agree non-trivially on some input, i.e., whether $u(\sigma)=$ $v(\sigma)$ for some non-empty $\sigma \in \Sigma^{+}$. This undecidable problem plays a central role in computer science because it is very often easier and more natural to prove undecidability by reduction from PCP than from, say, the halting problem for Turing machines.

In this paper we introduce PEP, a variant of PCP where one asks whether $u(\sigma)$ is a subword of $v(\sigma)$ for some $\sigma$. The subword relation, also called embedding, is denoted

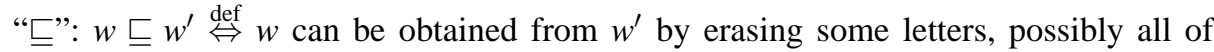
them, possibly none. We also introduce PEP ${ }^{\text {reg }}$, an extension of PEP where one adds the requirement that a solution $\sigma$ belongs to a regular language $R \subseteq \Sigma^{*}$.

As far as we know, PEP and PEP ${ }^{\text {reg }}$ have never been considered in the literature [13, 9]. This is probably because PEP is trivial (Prop. 3.1). However, and quite surprisingly, adding a regular constraint makes the problem considerably harder. In this paper we show that PEPreg is decidable but that it is not primitive recursive.

Channel systems. What led us to consider PEPreg are verification problems for channel systems, i.e., systems of finite-state machines that communicate asynchronously via unbounded FIFO channels. These systems are Turing-powerful in general but several restricted families or variants have decidable verification problems. For example lossy channel systems, where messages can be lost nondeterministically, have decidable reachability and termination problems $[7,3,15]$. For systems with one reliable channel (no message losses), reachability is easily decidable if the system is unidirectional: one

\footnotetext{
* Work supported by the Agence Nationale de la Recherche, grant ANR-06-SETIN-001.
} 

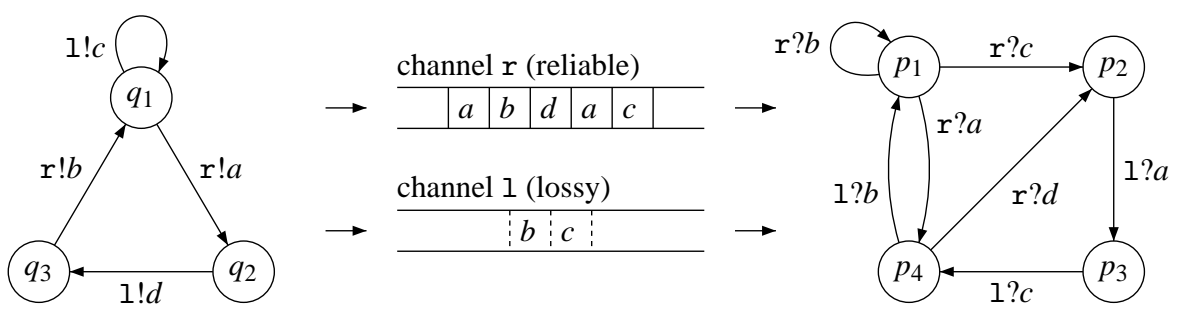

Fig. 1. A unidirectional channel system with one reliable and one lossy channel

sender sends messages to a receiver via the reliable channel, but no communication is possible in the other direction. With two (reliable) unidirectional channels between the sender and the receiver, reachability is undecidable. The open question that motivated our study is ReachUcs, i.e., reachability for channel systems with unidirectional communication through one reliable and one unreliable channels, as illustrated in Figure 1.

It is easy to reduce PEP and PEPreg to ReachUcs. It turns out that reductions from ReachUcs to PEP reg also exist. More surprisingly, we are able to reduce PEPreg to ReachLcs, the reachability problem for (classical) lossy channel systems, and to reduce ReachLcs to ReachUcs. Finally, all three problems are equivalent.

Summary of our contributions. 1. We introduce PEPreg, a new decidable variant of the $\mathrm{PCP}$ problem that is based on the subword relation. A surprising fact is that the regularity constraint makes PEPreg very different from PEP, and highly non-trivial.

2. We prove that PEPreg is equivalent to (i.e., inter-reducible with) ReachUcs and ReachLcs, two verification problems for systems of communicating automata. This provides the decidability of ReachUcs (and a new decidability proof for ReachLcs).

3. This shows that PEPreg is not primitive recursive (since ReachLcs is not either [15]).

This last point is quite interesting. In recent years, several problems coming from various areas have been shown to be not primitive recursive by reductions from ReachLcs: see, e.g., [2, 4, 6, 8, 10-12]. This is a clear indication that ReachLcs and equivalent problems occupy a specific niche that had not been identified previously. Discovering a simple and natural problem like PEPreg amid this class will help extend the range of problems that can be connected to the class: PEPreg can be used to simplify existing reduction proofs, and make some future proofs easier to obtain.

Outline of the paper. Section 2 recalls the necessary definitions and notations. We prove that PEPreg is decidable in Section 3 and explore variants and extensions in Section 4. The reductions between PEP ${ }^{\text {reg }}$ and ReachLcs or ReachUcs are given in sections 5 and 6. Proofs omitted for lack of space can be found in the long version of this paper [5].

\section{Notations and definitions}

Words. We write $u, v, w, t, \sigma, \rho, \alpha, \beta, \ldots$ for words, i.e., finite sequences of letters such as $a, b, i, j, \ldots$ from alphabets $\Sigma, \Gamma, \ldots$, and denote with $u . v$, or $u v$, the concatenation of $u$ and $v$. The length of $u$ is written $|u|$. A morphism from $\Sigma^{*}$ to $\Gamma^{*}$ is a map $h: \Sigma^{*} \rightarrow \Gamma^{*}$ that 
respects the monoidal structure, i.e., with $h(\varepsilon)=\varepsilon$ and $h(\sigma . \rho)=h(\sigma) \cdot h(\rho)$. A morphism $h$ is completely defined by its image $h(1), h(2), \ldots$, on $\Sigma=\{1,2, \ldots\}$. We often simply write $h_{1}, h_{2}, \ldots$, and $h_{\sigma}$, instead of $h(1), h(2), \ldots$, and $h(\sigma)$.

Quotients. Let $L$ be a language and $m$ a word: $m \backslash L \stackrel{\text { def }}{=}\{w \mid m . w \in L\}$ is the (right) quotient of $L$ by $m$. When $L \subseteq \Sigma^{*}$, we write $L(L)$ for the set $\left\{m \backslash L \mid m \in \Sigma^{*}\right\}$ of all quotients of $L$. It is well-known that if $R$ is a regular language, then $L(R)$ is finite and only contains regular languages (that still have their quotients in $L(R)$ ). $L(R)$ can be built effectively from a canonical DFA for $R$ just by varying the initial state.

Embeddings. Given two words $u=a_{1} \ldots a_{n}$ and $v=b_{1} \ldots b_{m}$, we write $u \sqsubseteq v$ when $u$ is a subword of $v$, i.e., when $u$ can be obtained by erasing some letters (possibly none) from $v$. For example, $a b b a \sqsubseteq a b r a c a d a b r a$. Equivalently, $u \sqsubseteq v$ when $u$ can be embedded in $v$, i.e., when there exists an order-preserving injective map $h:\{1, \ldots, n\} \rightarrow\{1, \ldots, m\}$ such that $a_{i}=b_{h(i)}$ for all $i=1, \ldots, n$. It is well-known that the subword relation is a partial ordering on words, and it is a well-quasi-ordering (Higman's Lemma) when we consider words over a fixed finite alphabet. This means that any set of words has a finite number of minimal elements (minimal w.r.t. $\sqsubseteq$ ).

Upward-closure. A language $L \subseteq \Gamma^{*}$ is upward-closed if $u \in L$ and $u \sqsubseteq v$ imply $v \in L$. It is downward-closed if its complement is upward-closed. Higman's Lemma entails that upward-closed languages (hence also downward-closed languages) are regular.

Splitting words. When $u \sqsubseteq v$, we write $v[u]$ for the longest $v_{1}$ such that $v$ is some $v_{0} \cdot v_{1}$ with $u \sqsubseteq v_{0}$. Hence $v[u]$ is the longest suffix of $v$ that can be retained if one has to remove some prefix containing $u$. Dually, for any $u$ and $v$, we write $u\{v\}$ for the shortest $u_{1}$, such that $u$ can be written as some $u_{0} \cdot u_{1}$ with $u_{0} \sqsubseteq v$. Hence $u\{v\}$ is the shortest suffix of $u$ that can be obtained if one may only remove prefixes that are contained in $v$. Observe that $u\{v\}$ is always defined while $v[u]$ is only defined when $u \sqsubseteq v$.

When reasoning about embedding and concatenation, a natural and simple tool is the following.

Lemma 2.1 (Simple Decomposition Lemma). If $u . w \sqsubseteq v . t$ then either $u \sqsubseteq v$ or $w \sqsubseteq t$.

However, Lemma 2.1 only works one way. For deeper analyses, we shall need the following more powerful tool.

Lemma 2.2 (Complete Decomposition Lemma).

$$
u . w \sqsubseteq v . t \text { if and only if }\left\{\begin{aligned}
u \sqsubseteq v \text { and } w \sqsubseteq v[u] . t \\
\text { or } u \nsubseteq v \text { and } u\{v\} . w \sqsubseteq t .
\end{aligned}\right.
$$

\section{PEP: Post correspondence with embedding}

The problem we are considering is a variant of Post correspondence problem where equality is replaced by embedding, and where an additional regular constraint is imposed over the solution. 


\section{Problem PEPreg}

Instance: Two finite alphabets $\Sigma$ and $\Gamma$, two morphisms $u, v: \Sigma^{*} \rightarrow \Gamma^{*}$, and a regular language $R \subseteq \Sigma^{*}$.

Answer: Yes if and only if there exists a $\sigma \in R$ such that $u_{\sigma} \sqsubseteq v_{\sigma}$.

In the above definition, the regular constraint applies to $\sigma$ but this is inessential and our results still hold when the constraint applies to $u_{\sigma}$, or $v_{\sigma}$, or both (see Section 4).

For complexity issues, we assume that the constraint $R$ in a PEPreg instance is given as a nondeterministic finite-state automaton (NFA) $\mathcal{A}_{R}$. By a reduction between two decision problems, we mean a logspace many-one reduction. We say two problems are equivalent when they are inter-reducible.

PEP is the special case of PEPreg where $R$ is $\Sigma^{+}$, i.e., where there are no constraints over the form of a non-trivial solution. As far as we know, PEP and PEP ${ }^{\text {reg }}$ have never been considered in the literature and this is probably because PEP is trivial:

Proposition 3.1. There is a $\sigma \in \Sigma^{+}$such that $u_{\sigma} \sqsubseteq v_{\sigma}$ if and only if there is some $i \in \Sigma$ such that $u_{i} \sqsubseteq v_{i}$.

This is a direct corollary of Lemma 2.1. A consequence is that PEP is decidable in deterministic logarithmic space.

Surprisingly, adding a regularity constraint makes the problem much harder, as will be proved later. As of now, we focus on proving the following main result.

Theorem 3.2 (Main Result). PEPreg is decidable.

In the rest of this section, we assume a given PEPreg instance made of $u, v: \Sigma^{*} \rightarrow \Gamma^{*}$ and $R \subseteq \Sigma^{*}$. We consider some $\mathcal{L}(R)$-indexed families of languages in $\Gamma^{*}$ :

Definition 3.3 (Blocking family). An $\mathcal{L}(R)$-indexed family $\left(A_{L}, B_{L}\right)_{L \in \mathcal{L}(R)}$ of languages in $\Gamma^{*}$ is a blocking family if for all $L \in \mathcal{L}(R)$ :

$$
\begin{aligned}
& \sigma \in L \text { and } \alpha \in A_{L} \text { imply } \alpha u_{\sigma} \square v_{\sigma}, \\
& \sigma \in L \text { and } \beta \in B_{L} \text { imply } u_{\sigma} \nsubseteq \beta v_{\sigma} .
\end{aligned}
$$

The terminology "blocking" comes from the fact that the $\alpha$ prefix "blocks" solutions in $L$ to $\alpha . u_{\sigma} \sqsubseteq v_{\sigma}$. For $B_{L}$, the situation is dual: adding $\beta \in B_{L}$ is not enough to allow solutions in $L$ to $u_{\sigma} \sqsubseteq \beta . v_{\sigma}$.

There is a largest blocking family, called the blocker languages, or blocker family, $\left(X_{L}, Y_{L}\right)_{L \in \mathcal{L}(R)}$, given by:

$$
\begin{gathered}
X_{L} \stackrel{\text { def }}{=}\left\{\alpha \in \Gamma^{*} \mid \alpha u_{\sigma} \nsubseteq v_{\sigma} \text { for all } \sigma \in L\right\}, \\
Y_{L} \stackrel{\text { def }}{=}\left\{\beta \in \Gamma^{*} \mid u_{\sigma} \nsubseteq \beta v_{\sigma} \text { for all } \sigma \in L\right\} .
\end{gathered}
$$

A blocking family provides information about the absence of solutions to several variants of our PEPreg instance. For example, the $u, v, R$ instance itself is positive iff $\varepsilon \notin X_{R}$ iff $\varepsilon \notin Y_{R}$.

For proving that a given family is blocking, we use a criterion called "stability". 
Definition 3.4 (Stable family). An $L(R)$-indexed family $\left(A_{L}, B_{L}\right)_{L \in \mathcal{L}(R)}$ of languages is stable iff, for all $L \in \mathcal{L}(R)$ :

1. $A_{L} \subseteq \Gamma^{*}$ is upward-closed and $B_{L} \subseteq \Gamma^{*}$ is downward-closed,

2. if $\varepsilon \in L$, then $\varepsilon \notin A_{L} \cup B_{L}$,

3. for all $i \in \Sigma$ and $\alpha \in A_{L}$ :

(a) if $\alpha . u_{i} \sqsubseteq v_{i}$ then $v_{i}\left[\alpha . u_{i}\right] \in B_{i \backslash L}$,

(b) if $\alpha . u_{i} \nsubseteq v_{i}$ then $\left(\alpha . u_{i}\right)\left\{v_{i}\right\} \in A_{i \backslash L}$,

4. for all $i \in \Sigma$ and $\beta \in B_{L}$ :

(a) if $u_{i} \sqsubseteq \beta . v_{i}$ then $\left(\beta . v_{i}\right)\left[u_{i}\right] \in B_{i \backslash L}$,

(b) if $u_{i} \nsubseteq \beta . v_{i}$ then $u_{i}\left\{\beta . v_{i}\right\} \in A_{i \backslash L}$.

Recall that $A_{L}$ and $B_{L}$, being respectively upward- and downward-closed, must be regular languages. Observe also that $\varepsilon \in B_{L}$ iff $B_{L} \neq \varnothing$, while $\varepsilon \in A_{L}$ iff $A_{L}=\Gamma^{*}$.

Proposition 3.5 (Soundness). A stable family is a blocking family.

Proof. Assume that $\left(A_{L}, B_{L}\right)_{L \in L(R)}$ is stable. We prove that it satisfies (B1) and (B2) by induction on the length of $\sigma$.

Base case: $\sigma=\varepsilon$. Hence $u_{\sigma}=v_{\sigma}=\varepsilon$. Assuming $\alpha u_{\sigma} \sqsubseteq v_{\sigma}$ requires $\alpha=\varepsilon$ but if $\sigma \in L$, stability implies that $\varepsilon \notin A_{L}$. $\sigma \in L$ also implies that $B_{L}$ is empty so that $u_{\sigma} \nsubseteq \beta v_{\sigma}$ is vacuously true.

Inductive case: assume that $\sigma$ is some $i . \rho$ with $i \in \Sigma$ and $\rho \in \Sigma^{*}$. Recall that $\sigma \in L$ iff $\rho \in i \backslash L$.

Let $\alpha \in A_{L}$. If $\alpha u_{i} \sqsubseteq v_{i}$, then $v_{i}\left[\alpha u_{i}\right] \in B_{i \backslash L}$ by stability. Hence $u_{\rho} \not\left(v_{i}\left[\alpha u_{i}\right]\right) v_{\rho}$ by ind. hyp. Then $\alpha u_{\sigma}=\alpha u_{i} u_{\rho} \nsubseteq v_{i} v_{\rho}=v_{\sigma}$ by Lemma 2.2. If, on the other hand, $\alpha u_{i} \not v_{i}$, then $\left(\alpha u_{i}\right)\left\{v_{i}\right\} \in A_{i \backslash L}$ by stability, hence $\left(\alpha u_{i}\right)\left\{v_{i}\right\} u_{\rho} \nsubseteq v_{\rho}$ by ind. hyp., entailing $\alpha u_{\sigma} \nsubseteq v_{\sigma}$ by Lemma 2.2.

For $\beta \in B_{L}$ the reasoning is similar. If $u_{i} \sqsubseteq \beta v_{i}$, then $\left(\beta v_{i}\right)\left[u_{i}\right] \in B_{i \backslash L}$ by stability, hence $u_{\rho} \nsubseteq\left(\beta v_{i}\right)\left[u_{i}\right] v_{\rho}$ by ind. hyp., hence $u_{\sigma}=u_{i} u_{\rho} \nsubseteq \beta v_{i} v_{\rho}=\beta v_{\sigma}$ by Lemma 2.2. If, on the other hand, $u_{i} \nsubseteq \beta v_{i}$, then $u_{i}\left\{\beta v_{i}\right\} \in A_{i \backslash L}$ by stability, hence $u_{i}\left\{\beta v_{i}\right\} u_{\rho} \nsubseteq v_{\rho}$ by ind. hyp., hence $u_{\sigma} \nsubseteq \beta v_{\sigma}$.

The criterion is also sufficient:

Proposition 3.6 (Completeness). The blocker family $\left(X_{L}, Y_{L}\right)_{L \in L(R)}$ is stable.

Proof. Clearly, as defined by (B3) and (B4) and for any $L \in \mathcal{L}(R), X_{L}$ is upward-closed and $Y_{L}$ is downward-closed. Similarly, $\varepsilon \notin X_{L}$ and $\varepsilon \notin Y_{L}$ when $\varepsilon \in L$.

It remains to check conditions 3 and 4 for stability. We consider four cases:

$3 a$ Assume that $\alpha u_{i} \sqsubseteq v_{i}$ for some $i$ in $\Sigma$ and some $\alpha$ in some $X_{L}$. If, by way of contradiction, we assume that $v_{i}\left[\alpha . u_{i}\right] \notin Y_{i \backslash L}$ then, by (B4), there is some $\rho \in i \backslash L$ such that $u_{\rho} \sqsubseteq v_{i}\left[\alpha . u_{i}\right] v_{\rho}$. Thus $\alpha u_{i} u_{\rho} \sqsubseteq v_{i} v_{\rho}$ by Lemma 2.2, i.e., $\alpha u_{\sigma} \sqsubseteq v_{\sigma}$ writing $\sigma$ for i.p. But, since $\sigma \in L$, this contradicts $\alpha \in X_{L}$.

$4 a$ A similar reasoning applies if we assume that $u_{i} \sqsubseteq \beta v_{i}$ for some $i$ in $\Sigma$ and some $\beta$ in some $Y_{L}$ while $\left(\beta v_{i}\right)\left[u_{i}\right] \notin Y_{i \backslash L}$ : we derive from (B4) that $u_{\rho} \sqsubseteq\left(\beta v_{i}\right)\left[u_{i}\right] v_{\rho}$ for some $\rho \in i \backslash L$. Hence $u_{i} u_{\rho} \sqsubseteq \beta v_{i} v_{\rho}$ by Lemma 2.2, a contradiction since i. $\rho \in L$. 
$3 b$ If we assume that $\alpha u_{i} \nsubseteq v_{i}$ for $\alpha \in X_{L}$ and $\left(\alpha u_{i}\right)\left\{v_{i}\right\} \notin X_{i \backslash L}$ then, by (B3), there is some $\rho \in i \backslash L$ s.t. $\left(\alpha u_{i}\right)\left\{v_{i}\right\} u_{\rho} \sqsubseteq v_{\rho}$. Then $\alpha u_{i} u_{\rho} \sqsubseteq v_{i} v_{\rho}$ by Lemma 2.2, a contradiction since $i . \rho \in L$.

$4 b$ Similarly, assuming that $u_{i} \nsubseteq \beta v_{i}$ while $u_{i}\left\{\beta v_{i}\right\} \notin A_{i \backslash L}$, we derive $\left(u_{i}\left\{\beta v_{i}\right\}\right) u_{\rho} \sqsubseteq$ $v_{i} v_{\mathrm{\rho}}$, i.e., $u_{i} u_{\mathrm{\rho}} \sqsubseteq \beta v_{i} v_{\rho}$, another contradiction.

Proposition 3.7 (Stability is decidable). It is decidable whether an $\mathcal{L}(R)$-indexed family $\left(A_{L}, B_{L}\right)_{L \in L(R)}$ of regular languages is a stable family.

Proof. We can assume that the $A_{L}$ and $B_{L}$ are given by DFA's. Conditions 1 and 2 of stability are easy to check.

For a given $i \in \Sigma$ and $L \in \mathcal{L}(R)$, checking condition $3 a$ needs only consider $\alpha$ 's that are shorter than $v_{i}$, which is easily done.

Checking condition $3 b$ is trickier. One way to do it is to consider the set of all $\alpha$ 's such that $\alpha u_{i} \nsubseteq v_{i}$. This is a regular set that can be obtained effectively. Then the set of all corresponding $\left(\alpha u_{i}\right)\left\{v_{i}\right\}$ is also regular and effective (see [5]) so that we can check that it is included in $A_{i \backslash L}$.

For condition $4 a$, and given some $L \in \mathcal{L}(R)$ and some $i \in \Sigma$, the set of all $\beta$ 's such that $u_{i} \sqsubseteq \beta v_{i}$ is regular and effective. One can then compute the corresponding set of all $\left(\beta v_{i}\right)\left[u_{i}\right]$, again regular and effective, and check inclusion in $B_{i \backslash L}$. The complement set of all $\beta$ 's such that $u_{i} \nsubseteq \beta v_{i}$ is also regular and effective, and one easily derives the corresponding $u_{i}\left\{\beta v_{i}\right\}$ 's (a finite set of suffixes of $u_{i}$ ), hence checking condition $4 b$.

Proof (of Theorem 3.2). Since PEPreg is r.e., it is sufficient to prove that it is also co-r.e. For this we observe that, by Propositions 3.5 and 3.6, a PEP ${ }^{\text {reg }}$ instance is negative if, and only if, there exists a stable family $\left(A_{L}, B_{L}\right)_{L \in \mathcal{L}(R)}$ satisfying $\varepsilon \in A_{R}$. One can effectively enumerate all families $\left(A_{L}, B_{L}\right)_{L \in \mathcal{L}(R)}$ of regular languages and check whether they are stable (Proposition 3.7) (and have $\varepsilon \in A_{R}$ ). If the PEPreg instance is negative, this procedure will eventually terminate, e.g., when it considers the blocker family.

Remark 3.8. Computing the blocker family for a negative PEPreg instance cannot be done effectively (this is a consequence of known results on lossy channel systems). Thus when the procedure described above terminates, there is no way to know that it has encountered the largest blocking family.

\section{Variants and extensions}

Short morphisms. $\mathrm{PEP}_{\leq 1}^{\text {reg }}$ is $\mathrm{PEP}^{\text {reg }}$ with the constraint that all $u_{i}$ 's and $v_{i}$ 's have length $\leq 1$, i.e., they must belong to $\Gamma \cup\{\varepsilon\}$.

Proposition 4.1. PEPreg reduces to $\mathrm{PEP}_{\leq 1}^{\mathrm{reg}}$.

Proof (Sketch). Let $u, v, R$ be a PEPreg instance. For all $i \in \Sigma$, write $u_{i}$ in the form $a_{i}^{1} \ldots a_{i}^{l_{i}}$ and $v_{i}$ in the form $b_{i}^{1} \ldots b_{i}^{m_{i}}$. Let $k=\max \left\{l_{i}, m_{i} \mid i \in \Sigma\right\}$. One builds a PEP $\mathrm{PP}_{\leq 1}^{\text {reg }}$ instance $u^{\prime}, v^{\prime}, R^{\prime}$ by letting $\Sigma^{\prime} \stackrel{\text { def }}{=} \Sigma \times\{1,2, \ldots, k\}, u^{\prime}(i, p) \stackrel{\text { def }}{=} a_{i}^{p}$ if $p \leq l_{i}$, and $u^{\prime}(i, p) \stackrel{\text { def }}{=} \varepsilon$ otherwise. Similarly, $v^{\prime}(i, p)$ is $v_{i}^{p}$, the $p$ th letter in $v_{i}$, or $\varepsilon$. We now let $R^{\prime} \stackrel{\text { def }}{=} h(R)$ where $h: \Sigma \rightarrow \Sigma^{\prime}$ is the morphism defined by $h(i)=(i, 1)(i, 2) \ldots(i, k)$. Finally $u^{\prime}, v^{\prime}, R^{\prime}$ is a $\mathrm{PEP}_{\leq 1}^{\mathrm{reg}}$ instance that is positive iff $u, v, R$ is positive. 
Constraining $u_{\sigma}$ and $v_{\sigma}$. PEP ${ }^{\mathrm{u}}{ }^{\mathrm{reg}}$ is like $\mathrm{PEP} \mathrm{P}^{\text {reg }}$ except that the constraint $R \subseteq \Gamma^{*}$ now applies to $u_{\sigma}$ : a solution is some $\sigma \in \Sigma^{*}$ with $u_{\sigma} \in R$ (and $\left.u_{\sigma} \sqsubseteq v_{\sigma}\right)$. Similarly, PEP ${ }^{\mathrm{v}}$ reg has the constraint apply to $v_{\sigma}$, while PEP ${ }^{\text {uv }}{ }^{\text {reg }}$ has two constraints, $R_{1}, R_{2} \subseteq \Gamma^{*}$, that apply to, respectively and simultaneously, $u_{\sigma}$ and $v_{\sigma}$.

Proposition 4.2. PEP ${ }^{\mathrm{uv}}{ }^{\mathrm{reg}}$ reduces to $\mathrm{PEP} \mathrm{P}^{\mathrm{reg}}$.

Proof. Let $u, v, R_{1}, R_{2}$ be a PEP ${ }^{\mathrm{uv} \_r e g}$ instance. Let $R \stackrel{\text { def }}{=} u^{-1}\left(R_{1}\right) \cap v^{-1}\left(R_{2}\right)$. (Recall that the image of a regular $R$ by an inverse morphism is regular and can easily be constructed from $R$.) By definition $\sigma \in R$ iff $u_{\sigma} \in R_{1}$ and $v_{\sigma} \in R_{2}$. Thus the PEP ${ }^{\text {reg }}$ instance $u, v, R$ is positive iff $u, v, R_{1}, R_{2}$ is.

Reductions exist in the other direction, as the next two propositions show.

Proposition 4.3. PEPreg reduces to $\mathrm{PEP}^{\mathrm{v}}{ }^{\mathrm{r}}$ reg.

Proof (Sketch). Let $u, v, R$ be a PEPreg instance. W.l.o.g., we may assume that $\Sigma \cap \Gamma=$ $\varnothing$. Define a PEP ${ }^{\mathrm{v}}$ reg instance $u^{\prime}, v^{\prime}, R^{\prime}$ by letting $v^{\prime}: \Sigma^{*} \rightarrow(\Gamma \cup \Sigma)^{*}$ be given by $v_{i}^{\prime} \stackrel{\text { def }}{=} i . v_{i}$ and keeping $u^{\prime}=u$ unchanged. Let $R^{\prime} \stackrel{\text { def }}{=} h^{-1}(R)$ where $h:(\Gamma \cup \Sigma)^{*} \rightarrow \Gamma^{*}$ is the erasing morphism that suppresses letters from $\Sigma$. Note that $v_{\sigma}^{\prime} \in R^{\prime}$ iff $\sigma=h\left(v_{\sigma}^{\prime}\right) \in R$, so that $u^{\prime}, v^{\prime}, R^{\prime}$ is a positive PEP ${ }^{\vee} \_$reg instance iff $u, v, R$ is a positive PEP ${ }^{\text {reg }}$ instance.

Proposition 4.4. $\mathrm{PEP}_{\leq 1}^{\mathrm{reg}}$ reduces to $\mathrm{PEP}^{\mathrm{u}}$ reg .

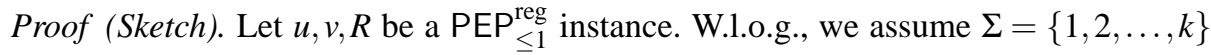
and let $\Sigma^{\prime} \stackrel{\text { def }}{=}\{0\} \cup \Sigma$ with $g: \Sigma^{\prime *} \rightarrow \Sigma^{*}$ the associated erasing morphism. We also assume $\Gamma \cap \Sigma^{\prime}=\varnothing$ and let $\Gamma^{\prime} \stackrel{\text { def }}{=} \Gamma \cup \Sigma^{\prime}$, with $h: \Gamma^{*} \rightarrow \Sigma^{*}$ as erasing morphism.

With $u, v, R$, we associate a PEP ${ }^{\text {u reg }}$ instance $u^{\prime}, v^{\prime}, R^{\prime}$ based on $\Sigma^{\prime}$ and $\Gamma^{\prime}$, and defined by $u_{0}^{\prime} \stackrel{\text { def }}{=} \varepsilon, v_{0}^{\prime} \stackrel{\text { def }}{=} 1.2 \ldots k$, and, for $i \in \Sigma, u_{i}^{\prime} \stackrel{\text { def }}{=} i . u_{i}$ and $v_{i}^{\prime} \stackrel{\text { def }}{=} v_{i}$. Letting $R^{\prime}=h^{-1}(R)$ ensures that $u_{\sigma}^{\prime} \in R^{\prime}$ iff $g(\sigma) \in R$. Clearly, if $u_{\sigma}^{\prime} \sqsubseteq v_{\sigma}^{\prime}$, then $u_{g(\sigma)} \sqsubseteq v_{g(\sigma)}$. Conversely, if $u_{\sigma^{\prime}} \sqsubseteq v_{\sigma^{\prime}}$, it is possible to find a $\sigma \in g^{-1}\left(\sigma^{\prime}\right)$ that satisfies $u_{\sigma}^{\prime} \sqsubseteq v_{\sigma}^{\prime}$ : this is just a matter of inserting enough 0 's at the appropriate places (and this is where we use the assumption that all $u_{i}$ 's and $v_{i}$ 's have length $\leq 1$ ).

Now, since PEP ${ }^{\mathrm{u} \_r e g}$ and $P E P^{\mathrm{v} \_ \text {reg }}$ are special cases of PEP ${ }^{\mathrm{uv} \_ \text {reg }}$, and since $P E P_{\leq 1}^{\text {reg }}$ is a special case of PEP ${ }^{\text {reg }}$, Propositions 4.1, 4.2, 4.3 and 4.4 entail the following.

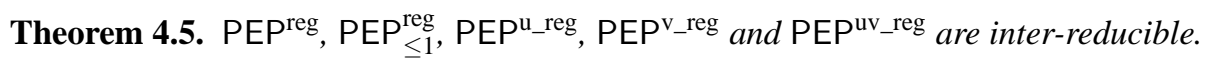

Context-free constraints and Presburger constraints. PEP ${ }^{\mathrm{cf}}$ is the extension of PEPreg where we allow the constraint $R$ to be any context-free language (say, given in the form of a context-free grammar). $\mathrm{PEP}^{\mathrm{dcf}}$ is $\mathrm{PEP} \mathrm{P}^{\mathrm{cf}}$ restricted to deterministic contextfree constraints. PEP ${ }^{\text {Pres }}$ is the extension where $R \subseteq \Sigma^{*}$ can be any language defined by a Presburger constraint over the number of occurrences of each letter from $\Sigma$ (or, equivalently, the commutative image of $R$ is a semilinear subset of the commutative monoid $\mathbb{N}^{\Sigma}$ ). 
Theorem 4.6. $\mathrm{PEP}^{\mathrm{dcf}}$, $\mathrm{PEP}^{\mathrm{cf}}$ and $\mathrm{PEP} \mathrm{P}^{\mathrm{Pres}}$ are undecidable.

Proof. The (classic) PCP problem reduces to PEP ${ }^{\text {dcf }}$ or PEP ${ }^{\text {Pres }}$ by associating, with an instance $u, v: \Sigma^{*} \rightarrow \Gamma^{*}$, the constraint $R_{\geq} \subseteq \Sigma^{+}$defined by

$$
\sigma \in R_{\geq} \stackrel{\text { def }}{\Leftrightarrow}\left|u_{\sigma}\right| \geq\left|v_{\sigma}\right| \text { and } \sigma \neq \varepsilon .
$$

Obviously, $u_{\sigma} \sqsubseteq v_{\sigma}$ and $\sigma \in R_{\geq}$iff $u_{\sigma}=v_{\sigma}$. Observe that $R_{\geq}$is easily defined in the quantifier-free fragment of Presburger logic. Furthermore, since $R_{\geq}$can be recognized by a counter machine with a single counter, it is indeed deterministic context-free.

\section{From PEPreg to lossy channel systems}

We now reduce PEPreg to ReachLcs, the reachability problem for lossy channel systems.

Systems composed of several finite-state components communicating via several channels (all of them lossy) can be simulated by systems with a single channel and a single component (see, e.g., [15, Section 5]). Hence we define here a lossy channel system (a LCS) as a tuple $S=(Q, \mathrm{M},\{\mathrm{c}\}, \Delta)$ where $Q=\left\{q_{1}, q_{2}, \ldots\right\}$ is a finite set of control states, $\mathrm{M}=\left\{a_{1}, a_{2}, \ldots\right\}$ is a finite message alphabet, $\mathrm{c}$ is the name of the single channel, and $\Delta=\left\{\delta_{1}, \ldots\right\}$ is the finite set of transition rules. Rules in $\Delta$ are writing rules, of the form $q \stackrel{\mathrm{c} ! u}{\longrightarrow} q^{\prime}$ (where $u \in \mathrm{M}^{*}$ is any sequence of messages), or reading rules $q \stackrel{\mathrm{c} ? u}{\longrightarrow} q^{\prime}$. We usually omit writing "c" in rules since there is only one channel, and no possibility for confusion.

The behaviour of $S$ is given in the form of a transition system. A configuration of $S$ is a pair $\langle q, v\rangle \in Q \times \mathrm{M}^{*}$ of a state and a channel contents. Transitions between configurations are obtained from the rules. Formally, $\langle q, v\rangle \rightarrow\left\langle q^{\prime}, v^{\prime}\right\rangle$ is a valid transition iff $\Delta$ contains a reading rule of the form $q \stackrel{? u}{\rightarrow} q^{\prime}$ and $v=u v^{\prime}$, or $\Delta$ contains a writing rule of the form $q \stackrel{! u}{\rightarrow} q^{\prime}$ and $v^{\prime}=v u^{\prime}$ for some $u^{\prime} \sqsubseteq u$. The intuition behind this definition is that a reading rule consumes $u$ from the head of the channel while a writing rule appends a (nondeterministically chosen) subsequence $u^{\prime}$ of $u$, and the rest of $u$ is lost. See, e.g., $[3,15]$ for more details on LCS's.

Remark 5.1. This behaviour is called write-lossy because messages can only be lost when they are appended to the channel, but once inside $c$ they remain there until a reading rule consumes them. This is different from, e.g., front-lossy semantics, where messages are lost when consumed (see [14]), or from the usual definition of LCS's, where messages can be lost at any time. These differences are completely inessential when one considers questions like reachability or termination, and authors use the definition that is technically most convenient for their purpose. In this paper, as in [1], the write-lossy semantics is the most convenient one.

Remark 5.2. Below we use extended rules of the form $q \stackrel{! u ? v}{\longrightarrow} q^{\prime}$. These are a shorthand notation for pairs of "consecutive" rules $q \stackrel{! u}{\rightarrow} s$ and $s \stackrel{? v}{\rightarrow} q^{\prime}$ where $s$ is an extra intermediary state that is not used anywhere else (and that we may omit listing in $Q$ ). 
ReachLcs, the reachability problem for LCS's, is the question, given a LCS $S$ and two states $q, q^{\prime} \in Q$, whether there exists a sequence of transitions in $S$ going from $\langle q, \varepsilon\rangle$ to $\left\langle q^{\prime}, \varepsilon\right\rangle$. The rest of this section proves the following theorem.

Theorem 5.3. PEPreg reduces to ReachLcs.

Remark 5.4. Since ReachLcs is decidable [3], Theorem 5.3 provides another proof that $\mathrm{PEP}^{\text {reg }}$ is decidable.

Let $u, v, R$ be a PEP ${ }^{\text {reg }}$ instance and $\sigma \in R$ be a solution. We say $\sigma$ is a direct solution if $u_{\rho} \sqsubseteq v_{\rho}$ for every prefix $\rho$ of $\sigma$. An equivalent formulation is: $\sigma=i_{1} \ldots i_{m}$ is a direct solution iff there are words $v_{1}^{\prime}, \ldots, v_{m}^{\prime}$ such that:

1. $v_{k}^{\prime} \sqsubseteq v_{i_{k}}$ for all $k=1, \ldots, m$,

2. $u_{i_{1}} \ldots u_{i_{m}}=v_{1}^{\prime} \ldots v_{m}^{\prime}$

3. $\left|u_{i_{1}} \ldots u_{i_{k}}\right| \leq\left|v_{1}^{\prime} \ldots v_{k}^{\prime}\right|$ for all $k=1, \ldots, m$.

A codirect solution is defined in a similar way, with the difference that we now require $\left|u_{i_{1}} \ldots u_{i_{k}}\right| \geq\left|v_{1}^{\prime} \ldots v_{k}^{\prime}\right|$ for all $k=1, \ldots, m$ (i.e., the $u_{i}^{\prime}$ 's are ahead of the $v_{i}^{\prime}$ s instead of lagging behind).

We let $P E P_{\text {dir }}^{\text {reg }}$ and $P E P_{\text {codir }}^{\text {reg }}$ denote the questions whether a PEP ${ }^{\text {reg }}$ instance has a direct (resp. codirect) solution. Obviously, $\mathrm{PEP}_{\mathrm{dir}}^{\mathrm{reg}}$ and $\mathrm{PEP}_{\text {codir }}^{\text {reg }}$ are equivalent problems since an instance $u, v, R$ has a codirect solution iff its mirror image $\widetilde{u}, \widetilde{v}, \widetilde{R}$ had a direct solution.

Proposition 5.5. $\mathrm{PEP}_{\text {dir }}^{\mathrm{reg}}$ (and $\mathrm{PEP}_{\text {codir }}^{\mathrm{reg}}$ ) reduce to ReachLcs.

Proof (Idea). Let $u, v, R$ be a PEP dir instance. Recall that $R$ is given via some NFA $\mathcal{A}_{R}=\left\langle Q, \Sigma, \delta, q_{\text {init }}, F\right\rangle$. With this instance, one associates a LCS $S=\langle Q, \Gamma,\{\mathrm{c}\}, \Delta\rangle$ with a graph structure $(Q, \Delta)$ inherited from $\mathcal{A}_{R}$. The difference is that an edge $r \stackrel{i}{\rightarrow} s$ in $\mathcal{A}_{R}$ gives rise to a transition rule $r \stackrel{! v_{i} ? u_{i}}{\longrightarrow} s$ in $S$. With such rules, $S$ can write the sequence $v_{1}^{\prime}, v_{2}^{\prime}, \ldots$ on $\mathrm{c}$, read $u_{i_{1}}, u_{i_{2}}, \ldots$ in lock-step fashion, and finally can move from the initial configuration $\left\langle q_{\text {init }}, \varepsilon\right\rangle$ to some final configuration $\langle f, \varepsilon\rangle$ with $f \in F$ iff the PEPreg instance has a direct solution. Restricting to direct solutions is what ensures that the $v_{1}^{\prime} \ldots v_{k}^{\prime}$ prefix that has been written on the channel is always longer than $u_{i_{1}} \ldots u_{i_{k}}$.

If we now look at a general solution to a PEPreg instance (more precisely a PEP ${ }_{\leq 1}^{\text {reg }}$ instance) it can be decomposed as a succession of alternating direct and codirect solutions to subproblems that are constrained by residuals of $R$.

Formally, assume $u, v, R$ is a $\mathrm{PEP}_{\leq 1}^{\mathrm{reg}}$ instance and $\sigma=i_{1} \ldots i_{m}$ is a solution. Then there are words $v_{1}^{\prime}, \ldots, v_{m}^{\prime}$ with $v_{k}^{\prime} \sqsubseteq v_{i_{k}}$ for $k=1, \ldots, m$, and such that $u_{i_{1}} \ldots u_{i_{m}}=$ $v_{1}^{\prime} \ldots v_{m}^{\prime}$. Now, for $0 \leq k \leq m$, define $d_{k} \stackrel{\text { def }}{=}\left|u_{i_{1}} \ldots u_{i_{k}}\right|-\left|v_{1}^{\prime} \ldots v_{k}^{\prime}\right|$. Then obviously $d_{0}=$ $d_{m}=0 . \sigma$ is a direct solution if $d_{k} \leq 0$ for all $k$. It is codirect if $d_{k} \geq 0$ for all $k$. In general, $d_{k}$ may oscillate between positive and negative values. But since all $u_{i}$ 's and $v_{i}$ 's have length $\leq 1$, the difference $d_{k+1}-d_{k}$ is in $\{-1,0,1\}$. Hence $d_{k}$ cannot change sign without being zero. In summary, the following holds:

Lemma 5.6. $A \mathrm{PEP}_{\leq 1}^{\mathrm{reg}}$ instance $u, v, R$ is positive iff there are states $q_{0}, q_{1}, \ldots, q_{2 m}$ in $\mathcal{A}_{R}$ with $q_{0}=q_{\mathrm{init}}, q_{2 m} \in F$, and such that, for all $0 \leq i<m, u, v, R_{2 i}$ is a positive $\mathrm{PEP}_{\mathrm{dir}}^{\mathrm{reg}}$ instance and $u, v, R_{2 i+1}$ is a positive $\mathrm{PEP}_{\text {codir }}^{\mathrm{reg}}$ instance (where $R_{i}$ is the regular language recognized by $\mathcal{A}_{R}$ when the initial state is changed to $q_{i}$ and the final states to $\left.\left\{q_{i+1}\right\}\right)$. 
With Lemma 5.6, one may prove Theorem 5.3 by extending the construction proving Proposition 5.5. Now the LCS looks for a sequence of alternating direct and codirect solutions. In direct mode, it proceeds as earlier until some state $q_{2 i+1}$ is reached. It may then switch to codirect mode. For this, it checks that the channel is empty (see below), guesses nondeterministically $q_{2 i+2}$, stores $q_{2 i+1}$ and $q_{2 i+2}$ in its finite memory, and now looks for a codirect solution to $u, v, R_{2 i+1}$. This is done by working on the mirror problem $\widetilde{u}, \widetilde{v}$, and moving backward from $q_{2 i+2}$ to $q_{2 i+1}$. When $q_{2 i+1}$ is reached (which can be checked since it has been stored when switching mode) it is possible to switch back to direct mode, starting from state $q_{2 i+2}$ (which was stored too), again after checking that the channel is empty. The emptiness checks use standard tricks, e.g., rules $q \stackrel{! ! ? \#}{\longrightarrow} q$ that write a special symbol $\# \notin \Gamma$ and consume it immediately.

\section{Reachability for unidirectional systems}

\subsection{Unidirectional systems}

ReachUcs is the reachability problem for UCS, i.e., systems of two components communicating unidirectionally via one reliable and one lossy channel, as illustrated in Fig. 1. A UCS has the form $S=\left(Q_{1}, Q_{2}, \mathrm{M},\{r, 1\}, \Delta_{1}, \Delta_{2}\right)$. The $Q_{1}, \Delta_{1}$ pair defines the sender component, with rules of the form $q \stackrel{\text { r!u }}{\longrightarrow} q^{\prime}$ or $q \stackrel{1 ! u}{\longrightarrow} q^{\prime}$. The $Q_{2}, \Delta_{2}$ pair has rules $q \stackrel{\text { r?u }}{\longrightarrow} q^{\prime}$ or $q \stackrel{1 ? u}{\longrightarrow} q^{\prime}$, defining the receiver component. A configuration is a tuple $\left\langle q_{1}, q_{2}, v_{1}, v_{2}\right\rangle$ with control states $q_{1}$ and $q_{2}$ for the components, contents $v_{1}$ for channel $r$, and $v_{2}$ for 1 .

The operational semantics is as expected. A rule $q \stackrel{\text { r!u }}{\longrightarrow} q^{\prime}$ (resp. $q \stackrel{1 ! u}{\longrightarrow} q^{\prime}$ ) from $\Delta_{1}$ gives rise to all transitions $\left\langle q, q_{2}, v_{1}, v_{2}\right\rangle \rightarrow\left\langle q^{\prime}, q_{2}, v_{1} u, v_{2}\right\rangle$ (resp. all $\left\langle q, q_{2}, v_{1}, v_{2}\right\rangle \rightarrow$ $\left\langle q^{\prime}, q_{2}, v_{1}, v_{2} u^{\prime}\right\rangle$ for $\left.u^{\prime} \sqsubseteq u\right)$. A rule $q \stackrel{\text { r?u }}{\longrightarrow} q^{\prime}$ (resp. $q \stackrel{1 ? u}{\longrightarrow} q^{\prime}$ ) from $\Delta_{2}$ gives rise to all transitions $\left\langle q_{1}, q, u v_{1}, v_{2}\right\rangle \rightarrow\left\langle q_{1}, q^{\prime}, v_{1}, v_{2}\right\rangle$ (resp. all $\left.\left\langle q_{1}, q, v_{1}, u v_{2}\right\rangle \rightarrow\left\langle q_{1}, q^{\prime}, v_{1}, v_{2}\right\rangle\right)$. Observe that message losses only occur when writing to channel 1 .

Remark 6.1. A consequence of unidirectionality is that a run $\left\langle q_{1}, q_{2}, v_{1}, v_{2}\right\rangle \rightarrow \cdots \rightarrow$ $\left\langle q_{1}^{\prime}, q_{2}^{\prime}, v_{1}^{\prime}, v_{2}^{\prime}\right\rangle$ can always be reordered so that it first uses only transitions from $\Delta_{1}$ that fill the channels, followed by only transitions from $\Delta_{2}$ that consume from them.

Theorem 6.2. [5] ReachLcs reduces to ReachUcs.

\subsection{From unidirectional systems to $P E P^{\text {reg }}$}

We now show that PEP ${ }^{\text {reg }}$ is expressive enough to encode ReachUcs.

Theorem 6.3. ReachUcs reduces to PEPreg.

Consider an ReachUcs instance that asks whether one can go from $\left\langle q_{0}, q_{0}^{\prime}, \varepsilon, \varepsilon\right\rangle$ to $\left\langle q_{f}, q_{f}^{\prime}, \varepsilon, \varepsilon\right\rangle^{1}$ in some UCS $S=\left(Q_{1}, Q_{2}, \mathrm{M},\{\mathrm{r}, 1\}, \Delta_{1}, \Delta_{2}\right)$. Without loss of generality,

\footnotetext{
${ }^{1}$ For simplification purposes, this proof considers ReachUcs instances where the channels are empty in the starting and ending configurations. This is no real loss of generality since the general ReachUcs problem easily reduces to the restricted problem.
} 
we assume that the rules in $S$ only read or write at most one message: formally, we write $M_{\varepsilon}$ for $M \cup\{\varepsilon\}$ and denote with $\alpha(\delta) \in M_{\varepsilon}$ (resp. $\beta(\delta) \in M_{\varepsilon}$ ) the messages that rule $\delta$ writes to, or reads from, $r$ (resp. 1). Observe that whether $\alpha(\delta)$ and $\beta(\delta)$ are read or written depends on whether $\delta$ belongs to $\Delta_{1}$ or $\Delta_{2}$. Observe also that there is at least one $\varepsilon$ among $\alpha(\delta)$ and $\beta(\delta)$.

Assume that the ReachUcs instance is positive and that a witness run $\pi$ first uses a sequence of rules $\delta_{1} \ldots \delta_{m} \in \Delta_{1}^{*}$, followed by a sequence $\gamma_{1} \ldots \gamma_{l} \in \Delta_{2}^{*}$ (this special form is explained in Remark 6.1). Then $\pi$ first writes $w=\alpha\left(\delta_{1}\right) \ldots \alpha\left(\delta_{m}\right)$ to $r$, then reads $w^{\prime}=\alpha\left(\gamma_{1}\right) \ldots \alpha\left(\gamma_{l}\right)$ from $r$, and we conclude that $w=w^{\prime}$. Simultaneously, it writes a subword $w^{\prime \prime}$ of $\beta\left(\delta_{1}\right) \ldots \beta\left(\delta_{m}\right)$ to 1 , and reads it in the form $\beta\left(\gamma_{1}\right) \ldots \beta\left(\gamma_{l}\right)$.

We are now ready to express this as a PEPreg problem. Let $\Sigma \stackrel{\text { def }}{=} \Delta_{1} \cup \Delta_{2}$ (assuming $\left.\Delta_{1} \cap \Delta_{2}=\varnothing\right)$ and $\Gamma \stackrel{\text { def }}{=} \mathrm{M}$. The morphisms are given by

$$
u(\delta) \stackrel{\text { def }}{=}\left\{\begin{array} { l l } 
{ \beta ( \delta ) } & { \text { if } \delta \in \Delta _ { 2 } , } \\
{ \varepsilon } & { \text { otherwise, } }
\end{array} \quad v ( \delta ) \stackrel { \text { def } } { = } \left\{\begin{array}{ll}
\beta(\delta) & \text { if } \delta \in \Delta_{1}, \\
\varepsilon & \text { otherwise }
\end{array}\right.\right.
$$

Now write $R_{1}$ for the set of all sequences $\delta_{1} \ldots \delta_{m} \in \Delta_{1}^{*}$ that form a connected path from $q_{0}$ to $q_{f}$ in $Q_{1}$, and $R_{2}$ for the set of all sequences $\gamma_{1} \ldots \gamma_{l} \in \Delta_{2}^{*}$ that form a connected path from $q_{0}^{\prime}$ to $q_{f}^{\prime}$ in $Q_{2}$. Let $R_{3}$ contains all rules $\delta \in \Delta_{1} \cup \Delta_{2}$ with $\alpha(\delta)=\varepsilon$, and all sequences $\delta$. $\gamma$ in $\Delta_{1} \Delta_{2}$ with $\alpha(\delta)=\alpha(\gamma) . R_{1}$ and $R_{2}$ are regular subsets of $\Gamma^{*}$, while $R_{3}$ is even finite.

We now let $R \stackrel{\text { def }}{=}\left(R_{1} \bowtie R_{2}\right) \cap R_{3}^{*}$, where $\bowtie$ denotes the shuffle of two languages (recall that this is regularity preserving). We conclude the proof of Theorem 6.3 with:

Lemma 6.4. [5] $u, v, R$ is a positive PEP ${ }^{\text {reg }}$ instance iff the ReachUcs instance is positive.

By combining with Theorems 6.3 and 6.2 we obtain the equivalence (inter-reducibility) of our three problems: PEPreg, ReachLcs and ReachUcs. This has two important new corollaries:

Corollary 6.5. ReachUcs is decidable (but not primitive recursive).

Corollary 6.6. $\mathrm{PEP} \mathrm{P}^{\mathrm{reg}}$ is (decidable but) not primitive recursive.

\section{Concluding remarks}

We introduced PEPreg, a variant of Post Correspondence Problem based on embedding (a.k.a. subword) rather than equality. Furthermore, a regular constraint can be imposed on the allowed solutions, which makes the problem non-trivial.

PEP ${ }^{\text {reg }}$ was introduced while considering ReachUcs, a verification problem for channel systems where a sender may send messages to a receiver through one reliable and one lossy channel, and where no communication is allowed in the other direction.

Our main results are (1) a non-trivial proof that PEPreg is decidable, and (2) three non-trivial reductions showing that PEP ${ }^{\text {reg }}$, ReachUcs and ReachLcs are equivalent. ReachLcs is the now well-known verification problem for lossy channel systems, where 
all channels are lossy but where no unidirectionality restriction applies. The equivalence between the three problems has two unexpected consequences: it shows that ReachUcs is decidable, and that PEPreg is not primitive recursive. We also show that (3) PEPreg and $P E P_{\text {dir }}^{\text {reg }}$, an important variant, are inter-reducible.

Beyond the applications to the theory of channel systems (our original motivation), the discovery of PEPreg is interesting in its own right. Indeed, in recent years the literature has produced many hardness proofs that rely on reductions from ReachLcs. We expect that such results, existing or yet to come, are easier to prove by reducing from PEP ${ }^{\text {reg }}$, or from PEP dir , than from ReachLcs.

\section{References}

1. P. A. Abdulla, C. Baier, S. Purushothaman Iyer, and B. Jonsson. Simulating perfect channels with probabilistic lossy channels. Information and Computation, 197(1-2):22-40, 2005.

2. P. A. Abdulla, J. Deneux, J. Ouaknine, and J. Worrell. Decidability and complexity results for timed automata via channel machines. In Proc. ICALP 2005, vol. 3580 of Lect. Notes Comp. Sci., pp. 1089-1101. Springer, 2005.

3. P. A. Abdulla and B. Jonsson. Verifying programs with unreliable channels. Information and Computation, 127(2):91-101, 1996.

4. R. Amadio and Ch. Meyssonnier. On decidability of the control reachability problem in the asynchronous $\pi$-calculus. Nordic Journal of Computing, 9(2):70-101, 2002.

5. P. Chambard and $\mathrm{Ph}$. Schnoebelen. Post embedding problem is not primitive recursive, with applications to channel systems. Research Report LSV-07-28, Lab. Specification and Verification, ENS de Cachan, Cachan, France, September 2007.

6. S. Demri and R. Lazić. LTL with the freeze quantifier and register automata. In Proc. LICS 2006, pp. 17-26. IEEE Comp. Soc. Press, 2006.

7. A. Finkel. Decidability of the termination problem for completely specificied protocols. Distributed Computing, 7(3):129-135, 1994.

8. D. Gabelaia, A. Kurucz, F. Wolter, and M. Zakharyaschev. Non-primitive recursive decidability of products of modal logics with expanding domains. Annals of Pure and Applied Logic, 142(1-3):245-268, 2006.

9. V. Halava, M. Hirvensalo, and R. de Wolf. Marked PCP is decidable. Theoretical Computer Science, 255(1-2):193-204, 2001.

10. B. Konev, F. Wolter, and M Zakharyaschev. Temporal logics over transitive states. In Proc. CADE 2005, vol. 3632 of Lect. Notes Comp. Sci., pp. 182-203. Springer, 2005.

11. S. Lasota and I. Walukiewicz. Alternating timed automata. In Proc. FOSSACS 2005, vol. 3441 of Lect. Notes Comp. Sci., pp. 250-265. Springer, 2005.

12. J. Ouaknine and J. Worrell. On the decidability and complexity of Metric Temporal Logic over finite words. Logical Methods in Comp. Science, 3(1):1-27, 2007.

13. K. Ruohonen. On some variants of Post's correspondence problem. Acta Informatica, 4(19):357-367, 1983.

14. $\mathrm{Ph}$. Schnoebelen. Bisimulation and other undecidable equivalences for lossy channel systems. In Proc. TACS 2001, vol. 2215 of Lect. Notes Comp. Sci., pp. 385-399. Springer, 2001.

15. Ph. Schnoebelen. Verifying lossy channel systems has nonprimitive recursive complexity. Information Processing Letters, 83(5):251-261, 2002. 